\title{
Review: inactivated vaccines provide the greatest protection against influenza in healthy people
}

\author{
Demicheli V, Rivetti D, Deeks JJ, et al. Vaccines for preventing influenza in healthy adults. Cochrane Database Syst Rev \\ 2001;(4):CD001269 (latest version 3 Aug 2001).

\section{QUESTION: What is the effectiveness of vaccines in preventing influenza in healthy adults?}

\section{Data sources}

Studies were identified by searching Medline (1966-97) with the terms influenza, route (oral), route (parenteral), and vaccine; searching EMBASE/Excerpta Medica (1990-97) and the Cochrane Controlled Trials Register; handsearching the journal Vaccine to 1997; scanning the bibliographies of articles; and contacting manufacturers of vaccines and authors of studies in the review.

\section{Study selection}

Studies were selected if they were quasi-randomised or randomised controlled trials (RCTs) that compared influenza vaccines with placebo, control vaccines, or no intervention or doses or schedules of influenza vaccine in healthy people 14 to 60 years of age and that assessed protection from exposure to naturally occurring influenza.

\section{Data extraction}

Data were extracted on study quality, participant characteristics, intervention, and outcomes. Outcomes included rates of influenza (clinically defined [unspecified], clinically defined on the basis of specific symptoms or signs [specified], and serologically confirmed), hospital admissions, working days lost, and adverse events.

\section{Main results}

20 trials were included (14 RCTs) evaluating 3 types of vaccine: live attenuated aerosol ( $\mathrm{n}=26$ 369), inactivated aerosol ( $\mathrm{n}=1506)$, and inactivated parenteral ( $\mathrm{n}=23$ 628). Live aerosol vaccines were not effective for preventing either type of clinically defined influenza (2 trials). Inactivated vaccines were effective in preventing influenza: both inactivated aerosol and inactivated parenteral vaccines prevented unspecified clinically defined influenza, and inactivated parenteral vaccines prevented specified clinically defined influenza (table). Live aerosol and inactivated parenteral vaccines prevented serologically confirmed influenza (table). No studies of inactivated aerosol reported serologically confirmed influenza. Vaccine and placebo did not differ for working days lost (3 trials), hospital admissions (1 trial), or complications
(2 trials). Local tenderness and soreness were increased in patients who received inactivated parenteral vaccine (table). No increase occurred in systemic myalgia, fever, or fatigue.

\section{Conclusion}

Inactivated parenteral vaccines are effective in preventing influenza in healthy adults.

\section{COMMENTARY}

Influenza vaccine is clearly cost effective in elderly people and those with chronic cardiac or respiratory disease. It reduces hospital admissions, respiratory mortality, and all cause mortality. ${ }^{1}$ Important potential benefits also exist in vaccinating healthy adults between 18 and 64 years of age against influenza, including reductions in influenza-like illness (ILI), avoidance of disruption in daily living, decreases in work absenteeism, and reduction in physician visits for ILI. ${ }^{2}$ From a societal perspective, the average costs range from a net saving of US $\$ 46.85$ per vaccinated person to a net cost of $\$ 11.17$ per person. The difference depends in part on the factors inserted in the sensitivity analyses and the range of the variable assumptions for vaccine efficacy, infectivity, and cost at different vaccination sites (ie, private office $v$ work site). ${ }^{2}{ }^{2}$ When little or no match exists between the vaccine and epidemic strain, the benefit is small to nil.

Demicheli et al show that an influenza vaccine is effective in reducing serologically confirmed cases of influenza A but is less effective in reducing cases of clinical influenza. However, because clinical influenza is a syndrome caused by several viruses, including respiratory syncytial, parainfluenza, and other viruses, efficacy using the outcome of clinical influenza will typically be underestimated; serological confirmation is a more reliable outcome.

This review confirms that the inactivated influenza vaccine is an effective method for preventing influenza in healthy adults. When the influenza vaccine supply permits, healthy young adults would benefit by receiving the inactivated influenza vaccine.

Peter A Gross, MD Hackensack University Medical Center Hackensack, New Jersey, USA

1 Gross PA, Hermogenes AW, Sacks HS, et al. The efficacy of influenza vaccine in the elderly: a meta-analysis and review of the literature. Ann Intern Med 1995;123:518-27.

2 Bridges CB, Thompson WW, Meltzer MI, et al. Effectiveness and cost-benefit of influenza vaccination of healthy working adults: a randomized controlled trial. JAMA 2000;284:1655-63.

3 Nichol KL. Cost-benefit analysis of a strategy to vaccinate healthy working adults against influenza. Arch Intern Med 2001;161:749-59.

Vaccines $v$ placebo for preventing influenza in healthy adults at mean 87 days*

\begin{tabular}{|c|c|c|c|c|c|}
\hline Outcomes & Comparisons & Weighted event rates & Number of trials & RRR (95\% Cl) & NNT (Cl) \\
\hline \multirow[t]{2}{*}{ CDI unspecified } & IA $v$ placebo & $7.2 \%$ v $20 \%$ & 1 & $65 \%$ (32 to 82 ) & 8 (4 to 63$)$ \\
\hline & IP $v$ placebo & $17 \%$ v $21 \%$ & 5 & $31 \%(5$ to 51$)$ & $25(13$ to 250$)$ \\
\hline CDI specified & IP $v$ placebo & $45 \%$ v $53 \%$ & 4 & $26 \%(1$ to 45$)$ & 13 (8 to 29$)$ \\
\hline \multirow[t]{3}{*}{$\mathrm{SCl}$} & LA $v$ placebo & $0.7 \%$ v $8.5 \%$ & 2 & $79 \%$ (44 to 92 ) & Not significant \\
\hline & IP $v$ placebo & $2.9 \%$ v $8.7 \%$ & 4 & $65 \%$ (44 to 79 ) & 18 (12 to 34$)$ \\
\hline & & & & RRI (Cl) & NNH (Cl) \\
\hline Local adverse events & IP $v$ placebo & $64 \%$ v $35 \%$ & 4 & $113 \%$ (35 to 236 ) & 4 (3 to 7$)$ \\
\hline
\end{tabular}

${ }^{*} \mathrm{CDI}=$ clinically defined influenza; $\mathrm{IA}=$ inactivated aerosol vaccine; $\mathrm{IP}=$ inactivated parenteral vaccine; $\mathrm{LA}=$ live aerosol vaccine; $\mathrm{SCl}=$ serologically confirmed influenza. Other abbreviations defined in glossary; RRR, RRI, NNT, NNH, and Cl calculated from data in article using random effects. 